\title{
A Subsampling and Interpolation Technique for Reversible Histogram Shift Data Hiding
}

\author{
Yih-Chuan Lin ${ }^{1}$, Tzung-Shian $\mathrm{Li}^{1}$, Yao-Tang Chang ${ }^{2}$, \\ Chuen-Ching Wang ${ }^{3}$, and Wen-Tzu Chen ${ }^{4}$ \\ ${ }^{1}$ Dept. of Computer Science and Information Engineering, National Formosa University, \\ Yunlin 63201 Taiwan \\ ${ }^{2}$ Dept. of Electro-Optical Science and Engineering, Kao Yuan University, Taiwan \\ ${ }^{3}$ Dept. of Electrical Engineering, National Changhua Univeristy of Education, Taiwan \\ ${ }^{4}$ Institute of Telecommunications Management, National Cheng Kung University, Taiwan \\ lyc@nfu.edu.tw
}

\begin{abstract}
This paper proposes a novel reversible data hiding algorithm for images, which the original host image can be exactly recovered from the marked image after the hidden data has been extracted. The proposed algorithm considers shifting the histogram of the difference values between the subsampled target pixel intensities and their interpolated counterparts to hide secret data. The shifting of the histogram of difference values is carried out by modifying the target pixel values. As compared to other schemes, the proposed method can make more utilization of the correlation between nearby pixels in an image via simple interpolation techniques to increase embedding capacity without sacrificing much distortion for data hiding. The reason of the feasibility is that the difference histogram derived in the paper renders so highly centralized distribution around zero that much more embedding capacity than before can be thus obtained. The experimental results demonstrate that the proposed method not only provides larger embedding capacity than other histogram shifting methods but also maintains a high visual quality. Moreover the computational complexity of the proposed method is low since only simple arithmetic computations are needed.
\end{abstract}

Keywords: Reversible Data Hiding, Histogram Shifting, Subsampling, Interpolation.

\section{Introduction}

Data hiding is a method that embeds useful information into a digital content (such as video, audio, image, electronic documents) and extracts it at a later time for one or more of the purposes of copy control, content authentication, distribution tracking, and so forth. In most cases, the marked image would experience some distortion due to the modification on pixels for hiding data and cannot be inverted back to the original version. However, in some applications, such as the fields of the law enforcement, medical or military imagery system, any permanent distortion induced to the original images by the data hiding techniques is intolerable. In such cases, the 
original images have to be recovered without any distortion after the extraction of the embedded data. Data hiding techniques satisfying these requirements are referred to as the so-called reversible data hiding.

According to what we known so far, three categories of reversible data hiding methods are available thus far in literature: (a) Difference expansion methods [1-4]: This type of methods uses the average and difference values between any two neighboring pixels in the host image, and expands the difference value to create the embedding space for hiding secret messages. But it does require keeping a large location map of data embedding for the lossless recovery of host image. Thus data compression technique is usually employed to alleviate this problem. (b) Histogram shifting methods [5-11]: Most of these methods need to compute the histogram of the whole image and manipulate the histogram to hide data. They are simple because it even doesn't need any compression technique. However, the capacity of this method is usually insufficient for most applications. (c) LSB (least significant bit) replacement methods $[12,13]$ : Techniques that use this type of methods usually hides the secret data bits in the LSB positions of the host pixel values; while record the original LSB as overhead for the exact recovery of host image. In general, they would need a lossless data compression method to offer additional space for increasing data hiding capacity.

Among these methods, the histogram shifting based methods have the advantages of less distortion and better image quality. Because it modifies the intensities of pixels slightly, it can produce very high peak signal-to-noise ratio (PSNR) after embedding secret data [5]. However, the embedding capacity that the histogram shifting methods can provide is not sufficient for most applications. The capacity of this method depends on the height of histogram maximum peak point. If the histogram distribution shape is highly centralized, i.e., the height of the maximum (or peak) point is raised significantly, the embedding capacity would become larger. Therefore, many researchers have tried various approaches to centralize the histogram distribution for maximizing the capacity of histogram shifting methods. For example, in 2009, Kim brings up a method which utilizes the differences between sub-images in a host image which are obtained by some sub-sampling techniques to raise the hiding capacity of histogram shifting. Since the histogram of the difference values becomes rather centralized, a good performance was obtained by this method $[8,11]$. Nevertheless, we think that much more correlation that exists between neighboring pixels in an image can be extracted to use for increasing lossless data hiding capacity. Therefore, while following Kim's sub-sampling method, interpolation techniques are added in the paper to produce more centralized histograms, in which more correlation between proximity pixels in an image is utilized by interpolation of neighboring pixels to reduce the difference value between a target pixel and its interpolated one. The secret data bits are embedded by shifting the histogram of the interpolated difference values, where each shift of histogram bin is carried out by the modification of the correspondent target pixel values in the host image. Incorporating the interpolation into the sub-sampling method, both the image quality and hiding capacity can be improved for most images. 
The rest of this paper is organized as follows. In Section 2, we explain the proposed methods, where linear and bilinear sub-sampling methods are presented. In Section 3, embedding and extracting processes are illustrated. Section 4 is devoted to show experimental results. Finally conclusions are drawn in Section 5.

\section{Proposed Subsampling and Interpolation Technique}

Fig. 1 depicts the components that comprise the proposed data hiding scheme. As shown in Fig. 1, the input host image $I$ is partitioned in one subsampled sub-image and several target sub-images by sub-sampling technique. For each target sub-image, an interpolated sub-image is computed by an interpolation scheme. Then the difference between the subsampled and interpolated sub-images is computed. For embedding secret data, we take histogram shifting method which is mentioned by other scholars to embed data into the histogram of the difference values. In this section, subsampling and interpolation technique would be described in details.

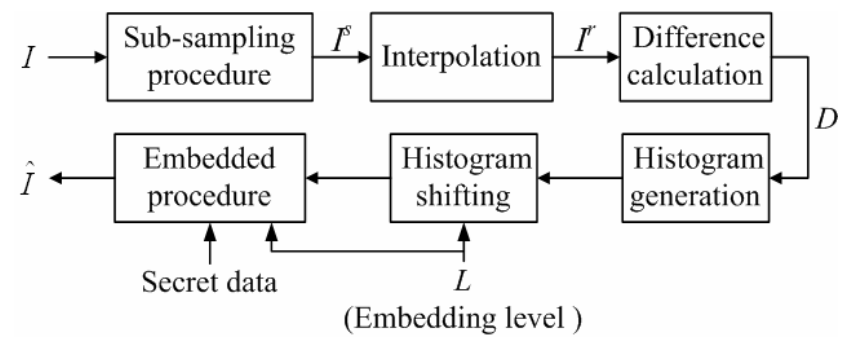

Fig. 1. Flowchart of data hiding process

For resizing an image, we usually prefer to use linear interpolation or bilinear interpolation. Kim's method [11] is equivalent to the simplest nearest neighbor interpolation, which relies solely on the difference between adjacent intensity. Thus the correlation between sub-images is not efficiently employed in his method. The reason is described as follows. Refer to Fig.2, where three consecutive pixels in an image is considered, and $b^{\prime}$ stands for the interpolated intensity for pixel $b$ by simple linear interpolation. Table 1 lists the statistical data on the different differences among the three considered pixels to show the advantage of linear interpolation technique. From Table 1, we can easily find that the distribution of difference values between $b$ and $b$ is more centralized than that of difference values between neighboring pixels

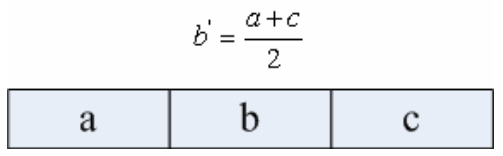

Fig. 2 Illustrative Example for linear interpolation 
Table 1. Comparison between the nearest neighbor and linear interpolation methods

\begin{tabular}{|l|l|l|l|l|}
\hline \multirow{2}{*}{} & $|l| l|l|$ & $\left|b^{\prime}-b\right|$ & \\
\cline { 2 - 5 } & $\mu$ & $\sigma$ & $\mu$ & $\sigma$ \\
\hline Lenna & 6.45 & 9.35 & 3.99 & 5.27 \\
\hline Baboon & 17.2 & 17.8 & 12.2 & 12.4 \\
\hline F-16 & 6.66 & 16 & 3.96 & 8.15 \\
\hline Goldhill & 7.22 & 9.12 & 5.28 & 6.41 \\
\hline
\end{tabular}

\subsection{The Linear Interpolation Sub-sampling Method}

Assume that the input image is a 8-bit $N_{1} \times N_{2}$ image $I(i, j)$, where $i=0, \ldots, N_{1}-1, j=0, \ldots, N_{2}-1$, we divide the image $I$ into one sub-sampled image $I^{s}\left(k_{1}, k_{2}\right)$ and one target image $I^{t}\left(k_{1}, k_{2}\right)$, as described in (1). Based on $I^{s}\left(k_{1}, k_{2}\right)$, the reference image $I^{r}\left(k_{1}, k_{2}\right)$ that is used as the interpolated version of the target image is obtained by the linear interpolation defined in (2).

$$
\begin{gathered}
I^{s}\left(k_{1}, k_{2}\right)=I\left(k_{1}, 2 k_{2}\right), \quad I^{t}\left(k_{1}, k_{2}\right)=I\left(k_{1}, 2 k_{2}+1\right) \\
0 \leq k_{1} \leq M, \quad 0 \leq k_{2} \leq\left\lfloor\frac{N}{2}\right\rfloor-1 \\
I^{r}\left(k_{1}, k_{2}\right)=\left\lfloor\frac{I^{s}\left(k_{1}, k_{2}\right)+I^{s}\left(k_{1}, k_{2}+1\right)}{2}\right\rfloor, \text { where } 0 \leq k_{1} \leq M, 0 \leq k_{2} \leq\left\lfloor\frac{N}{2}\right\rfloor-1
\end{gathered}
$$

Using the reference image, the difference image is formed using (3),

$$
D\left(k_{1}, k_{2}\right)=I^{r}\left(k_{1}, k_{2}\right)-I^{t}\left(k_{1}, k_{2}\right)
$$

\subsection{The Bilinear Interpolation Sub-sampling Method}

For increasing more capacity, we can extend the simple sub-sampling and linear interpolation method to the two-dimensional counterpart. Fig. 3 depicts the two-dimensional sub-sampling and interpolation pattern. By this pattern, there is one

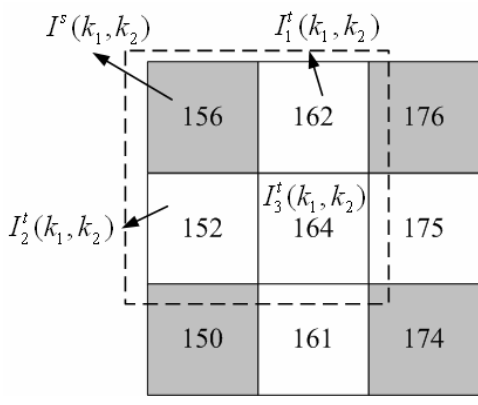

legend

sub-sampled

pixel

interpolated

pixel

Fig. 3. Example of bilinear interpolation method 
sub-sampled image $I^{s}\left(k_{1}, k_{2}\right)$ and three target images produced using (4). For each sub-sampled pixel, three correspondent interpolated pixels are calculated as the reference pixels according to (5). An index $q$ is used to specify which reference (target) is employed.

$$
\begin{aligned}
& I^{S}\left(k_{1}, k_{2}\right)=I\left(2 k_{1}, 2 k_{2}\right) \\
& I_{1}^{t}\left(k_{1}, k_{2}\right)=I\left(2 k_{1}, 2 k_{2}+1\right), \quad I_{2}^{t}\left(k_{1}, k_{2}\right)=I\left(2 k_{1}+1,2 k_{2}\right), I_{3}^{t}\left(k_{1}, k_{2}\right)=I\left(2 k_{1}+1,2 k_{2}+1\right) \\
& 0 \leq k_{1} \leq\left\lfloor\frac{M}{2}\right\rfloor-1, \quad 0 \leq k_{2} \leq\left\lfloor\frac{N}{2}\right\rfloor-1 \\
& I_{1}^{r}\left(k_{1}, k_{2}\right)=\left\lfloor\frac{I^{s}\left(k_{1}, k_{2}\right)+I^{s}\left(k_{1}, k_{2}+1\right)}{2}\right\rfloor, I_{2}^{r}\left(k_{1}, k_{2}\right)=\left\lfloor\frac{I^{s}\left(k_{1}, k_{2}\right)+I^{s}\left(k_{1}+1, k_{2}\right)}{2}\right\rfloor \\
& I_{3}^{r}\left(k_{1}, k_{2}\right)=\left\lfloor\frac{I^{s}\left(k_{1}, k_{2}\right)+I^{s}\left(k_{1}, k_{2}+1\right)+I^{s}\left(k_{1}+1, k_{2}\right)+I^{s}\left(k_{1}+1, k_{2}+1\right)}{4}\right\rfloor \\
& 0 \leq k_{1} \leq\left\lfloor\frac{M}{2}\right\rfloor-1, \quad 0 \leq k_{2} \leq\left\lfloor\frac{N}{2}\right\rfloor-1
\end{aligned}
$$

The difference images of bilinear interpolation is formed by (6).

$$
D_{q}\left(k_{1}, k_{2}\right)=I_{q}^{r}\left(k_{1}, k_{2}\right)-I_{q}^{t}\left(k_{1}, k_{2}\right) \quad 1 \leq q \leq 3
$$

\section{Embedding and Extracting Processes}

In this section, we describe the procedure that shifts the histogram of difference values and embeds data in the host image. The process is similar to other histogram shifting methods. Instead of directly shifting the difference values of histogram, the proposed method modifies the pixels in the target images to reflect the shifting for embedding operation. Because we have more than one target image in the bilinear method, we define a symbol $T$ to represent the number of target images used in embedding process. In linear interpolation situation, $T$ is set to 1 , and $T$ is set up to 3 in bilinear interpolation. The index of reference image or target image is less than $T$. The embedding level $L$ in the embedding process is pre-specified. If we want larger capacity, we set bigger $L$ and vice versa. We also denote $W B$ as the embedding secret data. When modifying the target images $I_{q}^{t}$ for embedding, we get the marked images $\hat{I}_{q}^{t}$. The embedding process is descried as follows:

Setp1: Shift the histogram outwards:

$$
\hat{I}_{q}^{t}\left(k_{1}, k_{2}\right)=\left\{\begin{array}{l}
I_{q}^{t}\left(k_{1}, k_{2}\right)+L+1 \text { if } D_{q}\left(k_{1}, k_{2}\right) \leq-L-1 \\
I_{q}^{t}\left(k_{1}, k_{2}\right)-L-1 \text { if } D_{q}\left(k_{1}, k_{2}\right) \geq L+1
\end{array} \quad \text { where } 1 \leq q \leq T\right.
$$


Step 2: Embed secret data $W B$ in the difference histogram.

$$
\hat{I}_{q}^{t}\left(k_{1}, k_{2}\right)= \begin{cases}I_{q}^{t}\left(k_{1}, k_{2}\right)-D_{q}\left(k_{1}, k_{2}\right)+W B, & \text { for }-L \leq D_{q}\left(k_{1}, k_{2}\right)<0 \\ I_{q}^{t}\left(k_{1}, k_{2}\right)-D_{q}\left(k_{1}, k_{2}\right)-W B, & \text { for } 0 \leq D_{q}\left(k_{1}, k_{2}\right) \leq L\end{cases}
$$

where $1 \leq q \leq T, W B \in\{0,1\}$

When the message is embedded into the target image or we shift the target image, over/underflow might occur. A modular addition with a cycle as that mentioned in [14] is employed. If a difference value is tested to be over/underflow as a result of hiding the secret data bit, the embedding operation has to be changed to use (9), instead of (8). In our experiments in the paper, the method uses an adaptive cycle of length to avoid a salt-and-pepper noise, as defined in (9). For example, the length $C$ of the cycle is set to 128 , just as the permutation of integers: $0 \leftrightarrow 1 \leftrightarrow \cdots \leftrightarrow 127 \leftrightarrow 0,128 \leftrightarrow$ $129 \leftrightarrow \cdots \leftrightarrow 255 \leftrightarrow 128$ for the image Lenna, when the maximum difference value between the sub-images is found to be 76 .

$$
\begin{aligned}
& \hat{I}_{q}^{t}\left(k_{1}, k_{2}\right)+i=C\left\lfloor\frac{I_{q}^{t}\left(k_{1}, k_{2}\right)}{C}\right\rfloor+\bmod \left(I_{q}^{t}\left(k_{1}, k_{2}\right)+i, C\right) \\
& \text { where } 1 \leq q \leq T, i= \begin{cases}D_{q}\left(k_{1}, k_{2}\right) \pm W B & \text { when }-L \leq D_{q}\left(k_{1}, k_{2}\right) \leq L \\
\pm(L+1) & \text { when } D_{q}\left(k_{1}, k_{2}\right)<-L-1 \text { or } D_{q}\left(k_{1}, k_{2}\right)>L+1\end{cases}
\end{aligned}
$$

In extracting process, we first generate the difference values from the marked image. Because the difference values are central to zero under normal circumstances, we can determine which target pixels have used modular addition if the difference values are very big. Thus we can restore these pixels back firstly. A similar approach is mentioned in [11].

The extracting and recovering processes are expressed as follows:

Step 1: Sub-sample the marked image using the same order as that used in the embedding procedure. Then we can compute the differences between the reference images and target images, and generate the histogram of difference values.

Step 2: Scan each of the difference images, if a difference value is within $[-2 L-1$, $2 L+1]$, the extract process is performed according to (10).

$$
W B=\left\{\begin{array}{l}
0, \text { if } D_{q}\left(k_{1}, k_{2}\right) \% 2=0 \\
1, \text { if } D_{q}\left(k_{1}, k_{2}\right) \% 2=1
\end{array} \text {, for }-2 L-1 \leq D_{q}\left(k_{1}, k_{2}\right) \leq 2 L+1\right.
$$

Step 3: Recover the target pixels if difference values within [-2L-1, 2L+1] using (11).

$$
\begin{aligned}
& I_{q}^{t}\left(k_{1}, k_{2}\right)=\left\{\begin{array}{ll}
\hat{I}_{q}^{t}\left(k_{1}, k_{2}\right)=\hat{I}_{q}^{t}\left(k_{1}, k_{2}\right)+l+1 & \text { if } D_{q}\left(k_{1}, k_{2}\right)=2 l+1 \\
\hat{I}_{q}^{t}\left(k_{1}, k_{2}\right)=\hat{I}_{q}^{t}\left(k_{1}, k_{2}\right)+l & \text { if } D_{q}\left(k_{1}, k_{2}\right)=2 l
\end{array} \text { for } 0 \leq l \leq L\right. \\
& I_{q}^{t}\left(k_{1}, k_{2}\right)=\left\{\begin{array}{ll}
\hat{I}_{q}^{t}\left(k_{1}, k_{2}\right)=\hat{I}_{q}^{t}\left(k_{1}, k_{2}\right)+l-1 & \text { if } D_{q}\left(k_{1}, k_{2}\right)=2 l-1 \\
\hat{I}_{q}^{t}\left(k_{1}, k_{2}\right)=\hat{I}_{q}^{t}\left(k_{1}, k_{2}\right)+l & \text { if } D_{q}\left(k_{1}, k_{2}\right)=2 l
\end{array} \text { for }-L \leq l<0\right.
\end{aligned}
$$

where $1 \leq q \leq T$ 
Step 4: Recover the target pixels if difference values outside $[-2 L-1,2 L+1]$ using (12).

$$
\begin{aligned}
& I_{q}^{t}\left(k_{1}, k_{2}\right)= \begin{cases}\hat{I}_{q}^{t}\left(k_{1}, k_{2}\right)+L+1, & \text { if } D_{q}\left(k_{1}, k_{2}\right)>2 L+1 \\
\hat{I}_{q}^{t}\left(k_{1}, k_{2}\right)-L-1, & \text { if } D_{q}\left(k_{1}, k_{2}\right)<-2 L-1\end{cases} \\
& \text { where } 0 \leq k_{1} \leq\left\lfloor\frac{M}{2}\right\rfloor-1,0 \leq k_{2} \leq\left\lfloor\frac{N}{2}\right\rfloor-1,1 \leq q \leq T
\end{aligned}
$$

With the embedding/extracting process pair, our proposed method is reversible, simple, and of less computational complexity. Besides, it doesn't have to transmit any overhead data because we solve the over/underflow problem by the modular method.

\section{Results}

Images of $512 \times 512 \times 8$ grayscale, as those shown in Fig. 4 , are used in the experiments for verifying the reversible data hiding algorithm proposed in this research. We have tested our proposed method in many kinds of images, such as nature images, map images, texture images, and satisfactory results are obtained.

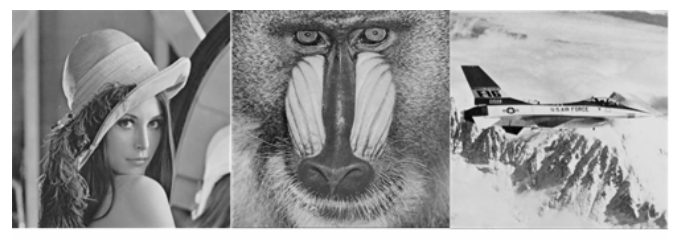

(a)

(b)

(c)

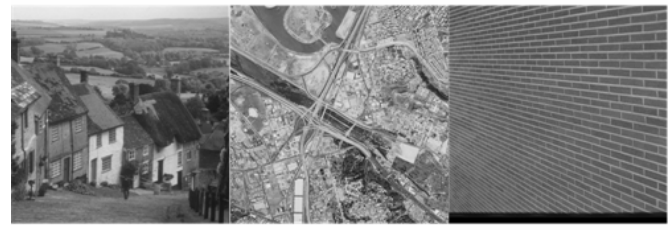

(d)

(e)

(f)

Fig. 4. Test image. (a) Lenna; (b) Baboon; (c) F16; (d) Goldhill; (e) Map; (f) Texture.

In Fig. 5, the original image Lenna and the marked images obtained by bilinear method are shown without prominent visual distortion. A comparison of capacity between Kim's algorithm and the proposed method is revealed in Fig.6. In Table 2 and Table 3, we not only compare the PSNR with that of Kim's method but also compare with that of Tian's method [1], which is very famous in data hiding field, after 50000 bits were embedded in the images considered.

Because the difference between the sub-images defined by our sub-sampling methods is small, over/underflow problem almost seldom happens. The proposed method is less likely to use modular addition and brings better visual quality. 


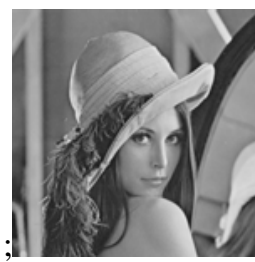

(a)

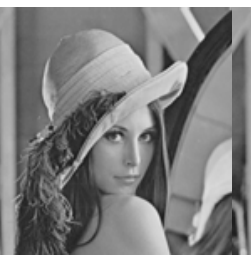

(b)

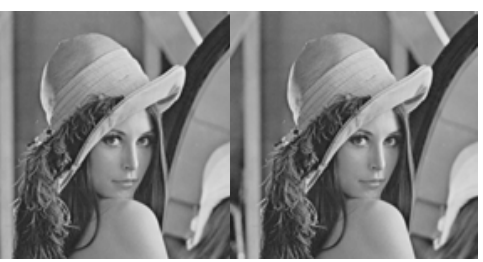

(c) (d)

Fig. 5. (a)original image; (b) 10000 bits hidden, PSNR=46.28 dB; (c)50000 bits hidden, $\mathrm{PSNR}=43.06 \mathrm{~dB}$; (d) 70000 bits hidden, $\mathrm{PSNR}=41.33 \mathrm{~dB}$.

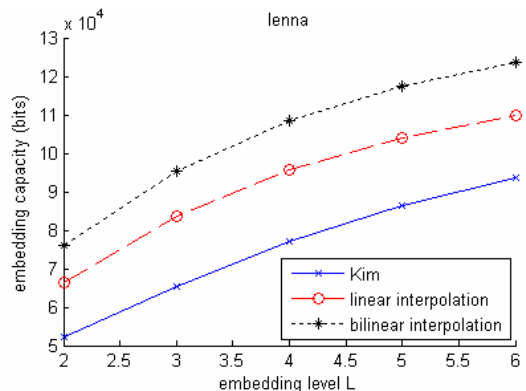

(a)

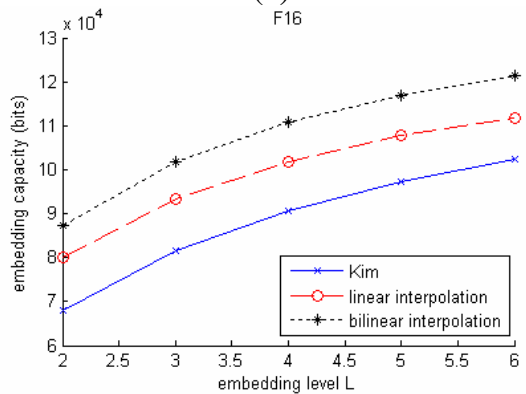

(c)

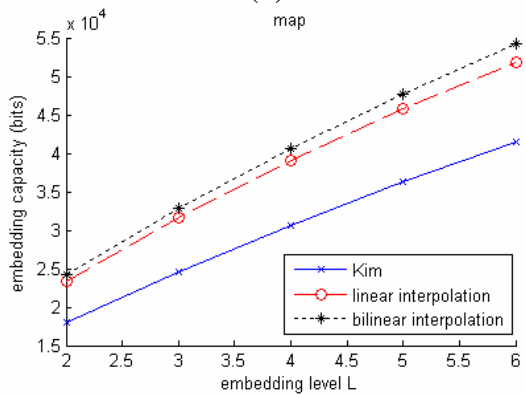

(e)

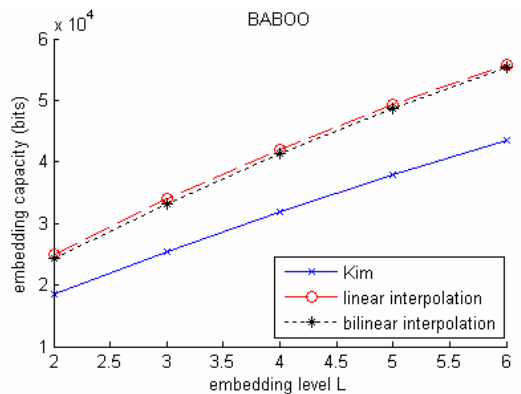

(b)

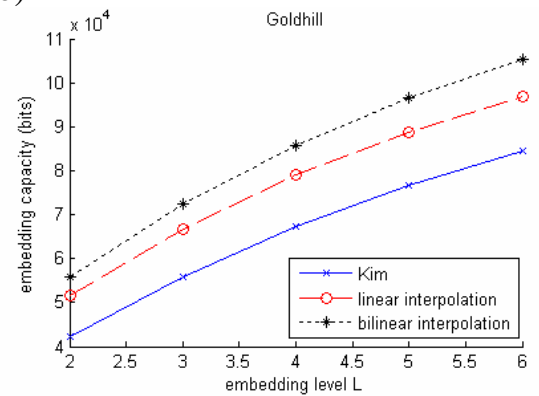

(d)

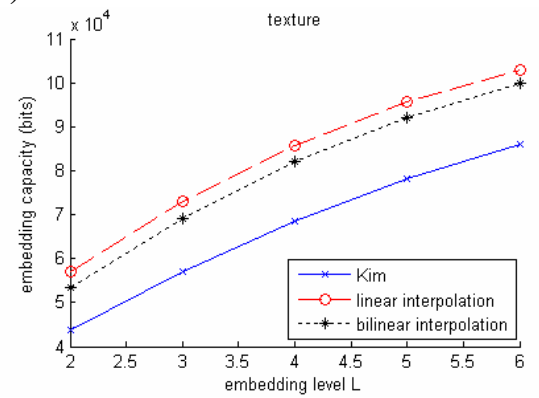

(f)

Fig. 6. Capacity comparison with varying embedding levels. (a) Lenna; (b) Baboon; (c) F-16; (d) Goldhill; (e) map; (f) texture. 
As shown in Fig.6, the worse capacity in Baboon and texture images for the bilinear interpolation method is resultant from the less correlation among the neighboring pixels in the column direction in the both images. If the sub-images subsampled by column direction are less correlated, the shape of difference histogram is more scattered and thus less capacity is gained. However the bilinear sub-sampling method is more adaptive. That is, if the images have stronger correlation in the column direction, the capacity of the proposed algorithm can be adjusted as demanded to very large when raising the value of embedding level $\mathrm{L}$.

Table 2. Comparison results in terms of PSNR(dB) for Lenna, Baboo and F16 images

\begin{tabular}{|l|l|l|l|}
\hline \multirow{2}{*}{} & \multicolumn{3}{|c|}{ PSNR(dB) } \\
\cline { 2 - 4 } & Lenna & Baboon & F16 \\
\hline Tian [1] & 37.89 & 28.51 & 37.50 \\
\hline Kim [8] & 42.76 & 33.30 & 43.01 \\
\hline Linear interpolation & 43.31 & 35.52 & 46.30 \\
\hline Bilinear interpolation & 45.63 & 34.93 & 43.24 \\
\hline
\end{tabular}

Table 3. Comparison results in terms of PSNR(dB) for Goldhill, map and texture images

\begin{tabular}{|l|l|l|l|}
\hline \multirow{2}{*}{} & \multicolumn{3}{|c|}{ PSNR(dB) } \\
\cline { 2 - 4 } & Goldhill & map & Texture \\
\hline Tian [1] & 38.68 & 29.33 & 37.94 \\
\hline Kim [8] & 40.50 & 33.42 & 40.58 \\
\hline Linear interpolation & 42.82 & 35.64 & 43.04 \\
\hline Bilinear interpolation & 42.36 & 35.10 & 40.38 \\
\hline
\end{tabular}

\section{Discussion and Conclusions}

As the results mentioned in Section 4, our proposed method has three strength points. First, it can provide more capacity than other methods. Second, it can keep relatively high image quality. Third, it is applicable to most types of image. In this research, the correlations between sub-images obtained by the proposed sub-sampling/interpolation technique are adequately utilized to increase the hiding capacity. The simulation results prove the effectiveness of the proposed method in improving the traditional histogram shifting in terms of both embedding capacity and objective fidelity. Furthermore, how to find other better ways defining subsample sub-image seems interesting in the future work.

\section{Acknowledgments}

This research was supported in part by National Science Council, Taiwan under research grants NSC 98-2221-E-150-051 and NSC 98-2221-E-244-002 to Yih-Chuan Lin and Yao-Tang Chang, respectively. 


\section{References}

1. Tain, J.: Reversible Data Embedding Using a Difference Expansion. IEEE transactions on circuits and systems for video technology 13(8) (2003)

2. Alattar, A.M.: Reversible watermarking using the difference expansion of a generalized integer transform. IEEE transactions on image processing 13(8) (2004)

3. Coltuc, D., Chassery, J.-M.: Very fast watermarking by reversible contrast mapping. IEEE Signal Processing Letters 14(4), 255-258 (2006)

4. Lin, C.-C., Yang, S.-P., Hsueh, N.-L.: Lossless data hiding based on difference expansion without a location map. In: IEEE Congress on Image and Signal Processing (2008)

5. Ni, Z., Shi, Y.-Q., Ansari, N., Su, W.: Reversible Data Hiding. IEEE Transactions on Circuits and Systems Video Technology 16(3) (2006)

6. Hwang, J.H., Kim, J.W., Choi, J.U.: A reversible watermarking based on histogram shifting. In: Shi, Y.Q., Jeon, B. (eds.) IWDW 2006. LNCS, vol. 4283, pp. 348-361. Springer, Heidelberg (2006)

7. Kuo, W.-C., Jiang, D.-J., Huang, Y.-C.: A reversible data hiding based on block division. In: Congress on image and signal processing, vol. 1, pp. 365-369 (2008)

8. Kim, K.-S., Lee, M.-J., Suh, Y.-H.: Histogram-based reversible data hiding technique using subsampling. In: Proceeding of the 10th ACM workshop on multimedia and security (September 2008)

9. Chung, K.-L., Huang, Y.-H., Yang, W.-N., Hsu, Y.-C.: Capacity maximization for reversible data hiding based on dynamic programming approach. Applied mathematics and computation, 284-292 (2009)

10. Lin, Y.-C., Li, T.-S.: High capacity histogram shifting lossless data hiding. In: IPPR conference on Computer Vision, Graphics, and Image Processing (2009)

11. Kim, K.-S., Leeand, M.-J., Suh, Y.-H.: Reversible data exploiting spatial correlation between sub-sampled images. Pattern Recognition 42, 3083-3096 (2009)

12. Celik, M.U., Sharma, G., Tekalp, A.M., Saber, E.: Lossless Generalized-LSB Data Embedding. IEEE transactions on image processing 14(2) (2005)

13. Ohyama, S., Niimi, M., Yamawaki, K., Noda, H.: Lossless data hiding using bit-depth embedding for JPEG2000 compressed bit stream. In: IEEE International Conference on Intelligent Information Hiding and Multimedia Signal Processing (2008)

14. Fridrich, J., Goljan, J., Du, R.: Invertible authentication. In: Proceedings of the SPIE, Security and Watermarking of Multimedia Contents, vol. 4314, pp. 197-208 (2001) 